\title{
Point and interval estimation for a simple step-stress model with Type-I censored data from geometric distribution
}

\author{
Ahmad Arefi ${ }^{a}$, Mostafa Razmkhah ${ }^{1, a}$ \\ ${ }^{a}$ Department of Statistics, Ferdowsi University of Mashhad, Iran
}

\begin{abstract}
The estimation problem of expected time to failure of units is studied in a discrete set up. A simple stepstress accelerated life testing is considered with a Type-I censored sample from geometric distribution that is a commonly used distribution to model the lifetime of a device in discrete case. Maximum likelihood estimators as well as the associated distributions are derived. Exact, approximate and bootstrap approaches construct confidence intervals that are compared via a simulation study. Optimal confidence intervals are suggested in view of the expected width and coverage probability criteria. An illustrative example is also presented to explain the results of the paper. Finally, some conclusions are stated.
\end{abstract}

Keywords: accelerated life testing, expected time to failure, Fisher information, maximum likelihood estimator, bootstrap sample

\section{Introduction}

There are situations in reliability and survival analysis for which the experiment may not terminate at an adequate time under normal conditions. In such situations, accelerated life testing experiments have been offered to obtain adequate life data. See, for example, Bagdonavicius and Nikulin (2002) and Nelson (1990). A special class of accelerated life testing is the step-stress testing for which the stress levels of the experiment change at some pre-specified times. Balakrishnan and Xie (2007a) obtained similar results when Type-I hybrid censored sample from exponential distribution is considered. Analogous work has been done under Type-II hybrid censoring by Balakrishnan and Xie (2007b). Balakrishnan et al. (2009) derived exact inference for a simple step-stress model from the exponential distribution when there is a time constraint on the duration of the experiment. See also, Balakrishnan and Han (2008) and Han and Balakrishnan (2010) for simple step-stress models under Type-II and Type-I censoring schemes, respectively.

In some situations, the life testing experiment must be investigated in a discrete setup. For example, suppose for example that the lifetimes of the units in an experiment depend on the number of times the units are switched on and off, the number of pages a printer prints, or the number of rotations of a machine. Let $w$ be the number of aforementioned shocks the units receive until they fail, so, $w$ is considered as the associated failure time. Censored samples in discrete setup have been studied by some authors. For example, Rezaei and Arghami (2002) investigated Type-I and Type-II

\footnotetext{
${ }^{1}$ Corresponding author: Department of Statistics, Faculty of Mathematical Sciences, Ferdowsi University of Mashhad,

P. O. Box 1159, Mashhad 91775, Iran. E-mail: razmkhah_m@um.ac.ir
}

Published 31 January 2017 / journal homepage: http://csam.or.kr

(c) 2017 The Korean Statistical Society, and Korean International Statistical Society. All rights reserved. 
right censoring in a discrete life model. Davarzani and Parsian (2011) considered discrete middle censored samples and used both classical and Bayesian approaches to do inference about the parameter of interest. Balakrishnan et al. (2011) studied characterizations of geometric distribution based on some properties of progressively Type-II right censored order statistics.

Suppose $n$ identical units are simultaneously placed on a test under an initial stress level $s_{0}$ and the stress levels are increased to $s_{1}, \ldots, s_{m}$ at pre-fixed integer numbers $w_{1}<\cdots<w_{m}$. If the units are subjected to shocks repeatedly $w_{m+1}$ times, where $w_{m+1}$ is a pre-fixed integer number, Type-I censoring scheme has been performed. Under this scheme, failure times greater than $w_{m+1}$ are not observed. In this paper, a simple step-stress scheme with only two stress levels $s_{0}$ and $s_{1}$ under Type-I censoring is considered. Assuming the failure times in each level of stress are geometrically distributed, Arefi and Razmkhah (2013) obtained the optimal time to change the stress level. Under the same assumptions, the present paper uses a classical approach to investigate the point and interval estimates of the expected times to failure, which are useful for reliability engineers. The performance of the proposed estimators in this paper may be improved when the optimal change times are used. Analogous work has been done by Arefi et al. (2011) in a Bayesian approach. Wang et al. (2012) investigated the parameter inference in step-stress accelerated life tests under a Type-II censoring model with geometric distribution.

The rest of this paper is as follows. The model considered in the paper is discussed in detail in Section 2. The maximum likelihood estimators (MLEs) of the parameters of interest are also obtained and the conditions for their existence are stated. The conditional distributions of the MLEs are investigated in Section 3. In Section 4, several methods are used to construct confidence intervals (CIs) for the unknown parameters. In Section 5, a simulation study is done to compare various CIs. In Section 7, some conclusions are stated and suggestions are made for applying suitable CIs.

\section{Model description and MLE}

Suppose $n$ identical units are simultaneously placed on a test under an initial stress level $s_{0}$ and the stress level increases to $s_{1}$ at the pre-fixed integer number $w_{1}$. Moreover, assume that the failure times of the units in stress levels $s_{0}$ and $s_{1}$ are geometrically distributed with success probabilities $p_{1}$ and $p_{2}$, respectively. That is, the cumulative distribution function (cdf) is given by

$$
F_{k}\left(x ; p_{k}\right)=1-q_{k}^{x}, \quad x=1,2, \ldots,
$$

where $q_{k}=1-p_{k}(k=1,2)$. Using the memoryless property of geometric distribution, it can be shown that the probability mass function (pmf) of the failure times in the simple step-stress model is as follows

$$
g(x)=\left\{\begin{array}{l}
g_{1}(x)=p_{1} q_{1}^{x-1}, \quad x=1,2, \ldots, w_{1}, \\
g_{2}(x)=p_{2} q_{1}^{w_{1}} q_{2}^{x-\left(w_{1}+1\right)}, \quad x=w_{1}+1, w_{1}+2, \ldots
\end{array}\right.
$$

Using (2.1), the corresponding cdf is

$$
G(x)=\left\{\begin{array}{l}
G_{1}(x)=1-q_{1}^{x}, \quad x=1,2, \ldots, w_{1}, \\
G_{2}(x)=1-q_{1}^{w_{1}} q_{2}^{x-w_{1}}, \ldots x=w_{1}+1, w_{1}+2, \ldots,
\end{array}\right.
$$

(see also, Arefi et al., 2011). Now, assume that the step-stress testing terminates when the units are subject to shocks repeatedly $w_{2}$ times, where $w_{2}$ is a pre-fixed integer number. Moreover, let $Z_{i}$ be the 
number of failures occur at time $i\left(i=1,2, \ldots, w_{2}\right)$. Rezaei and Arghami (2002) showed that the joint distribution of the $Z_{1}, \ldots, Z_{w_{2}}$ is multinomial with parameters $n, \pi_{1}, \ldots, \pi_{w_{2}+1}$, where

$$
\pi_{i}=\left\{\begin{array}{l}
p_{1} q_{1}^{i-1}, \quad i=1,2, \ldots, w_{1}, \\
p_{2} q_{1}^{w_{1}} q_{2}^{i-\left(w_{1}+1\right)}, \quad i=w_{1}+1, \ldots, w_{2}, \\
1-\sum_{i=1}^{w_{2}} \pi_{i}=q_{1}^{w_{1}} q_{2}^{w_{2}-w_{1}}, \quad i=w_{2}+1 .
\end{array}\right.
$$

That is,

$$
\begin{aligned}
P\left(Z_{1}=z_{1}, \ldots, Z_{w_{2}}=z_{w_{2}}\right)= & \frac{n !}{z_{1} ! \ldots z_{w_{2}} !\left(n-\sum_{i=1}^{w_{2}} z_{i}\right) !} \pi_{1}^{z_{1}} \ldots \pi_{w_{2}}^{z_{w_{2}}} \pi_{w_{2}+1}^{n-\sum_{i=1}^{w_{2}} z_{i}} \\
= & \frac{n !}{z_{1} ! \ldots z_{w_{2}} !\left(n-\sum_{i=1}^{w_{2}} z_{i}\right) !} p_{1}^{\sum_{i=1}^{w_{1}} z_{i}} q_{1}^{\sum_{i=1}^{w_{1}}(i-1) z_{i}+w_{1}\left(n-\sum_{i=1}^{w_{1}} z_{i}\right)} \\
& \times p_{2}^{\sum_{i=w_{1}+1}^{w_{2}} z_{i}} q_{2}^{\sum_{i=w_{1}+1}^{w_{2}}\left(i-w_{1}-1\right) z_{i}+\left(w_{2}-w_{1}\right)\left(n-\sum_{i=1}^{w_{2}} z_{i}\right)} .
\end{aligned}
$$

This paper infers on the expected time to failure of the products with geometric distributions in both levels of stress, i.e., $\theta_{1}=1 / p_{1}$ and $\theta_{2}=1 / p_{2}$. Using (2.4), the MLEs of $\theta_{1}$ and $\theta_{2}$ are readily obtained as

$$
\hat{\theta}_{1}=\frac{\sum_{i=1}^{w_{1}} i Z_{i}+w_{1}\left(n-R_{1}\right)}{R_{1}}
$$

and

$$
\hat{\theta}_{2}=\frac{\sum_{i=w_{1}+1}^{w_{2}} i Z_{i}-w_{1} R_{2}+\left(w_{2}-w_{1}\right)\left(n-R_{1}-R_{2}\right)}{R_{2}},
$$

respectively, where $R_{1}=\sum_{i=1}^{w_{1}} Z_{i}$ and $R_{2}=\sum_{i=w_{1}+1}^{w_{2}} Z_{i}$ represent the number of failures occur at stress levels $s_{0}$ and $s_{1}$, respectively. From (2.5) and (2.6), it is observed that the estimates of $\theta_{1}$ and $\theta_{2}$ are finite, when $R_{1} \neq 0$ and $R_{2} \neq 0$. Therefore, we consider the event

$$
A=\left\{1 \leq R_{1} \leq n-1,1 \leq R_{2} \leq n-R_{1}\right\},
$$

which ensures existence of $\hat{\theta}_{1}$ and $\hat{\theta}_{2}$. Note that the estimators proposed by Arefi and Razmkhah (2013) were constructed based on order statistics that needed overly heavy computations. But, the MLEs in (2.5) and (2.6) accelerate the computations. In the next section, the conditional cdfs of $\hat{\theta}_{1}$ and $\hat{\theta}_{1}$ are determined when event $A$ occurs.

Remark 1. In this paper, we have not assumed any relationships between the two stress levels. In fact, we assume that $\theta_{1}>\theta_{2}$ or equivalently $p_{1}<p_{2}$. Under this circumstance, it is emphasized that the expected lifetime at the higher stress level $s_{1}$ is less than the initial stress level $s_{0}$. In this way, the effect of accelerating stress on distribution of the lifetime is determined. In some situations, we may know that some particular relationships hold between the stress levels or parameters; for instance, $\theta_{2}=\lambda \theta_{1}$ with known $\lambda(0<\lambda<1)$. In such situations, it is adequate to estimate only $\theta_{1}$. Moreover, one can also use the likelihood ratio test to test the hypothesis $H_{0}: \theta_{2}=\lambda \theta_{1}$ for a specified $\lambda$. See, Balakrishnan and Han (2008), Balakrishnan and Xie (2007a, 2007b), Balakrishnan et al. (2009). 


\section{Conditional distributions of MLEs}

In this section, the conditional cdfs of the MLEs $\hat{\theta}_{1}$ and $\hat{\theta}_{2}$ are determined and used to find the exact CIs in the next sections. First, note that from (2.4), the following results deduce:

- The random variables $R_{1}$ and $R_{2}$ have trinomial distribution with pmf

$$
P\left(R_{1}=r_{1}, R_{2}=r_{2}\right)=c_{r_{1}, r_{2}} \beta_{1}^{r_{1}} \beta_{2}^{r_{2}} \beta_{3}^{n-r_{1}-r_{2}},
$$

where $0 \leq r_{1} \leq n, 0 \leq r_{2} \leq n-r_{1}$ and

$$
\begin{aligned}
c_{r_{1}, r_{2}} & =\frac{n !}{r_{1} ! r_{2} !\left(n-r_{1}-r_{2}\right) !}, \\
\beta_{1} & =\sum_{i=1}^{w_{1}} \pi_{i}=1-q_{1}^{w_{1}}, \\
\beta_{2} & =\sum_{i=w_{1}+1}^{w_{2}} \pi_{i}=q_{1}^{w_{1}}\left(1-q_{2}^{w_{2}-w_{1}}\right), \\
\beta_{3} & =1-\beta_{1}-\beta_{2}=q_{1}^{w_{1}} q_{2}^{w_{2}-w_{1}} .
\end{aligned}
$$

Note that the above notations are used throughout the paper. Using (2.7) and (3.1), we have

$$
\begin{aligned}
P(A) & =\sum_{r_{1}=1}^{n-1} \sum_{r_{2}=1}^{n-r_{1}} P\left(R_{1}=r_{1}, R_{2}=r_{2}\right) \\
& =1-\left(1-\beta_{1}\right)^{n}-\left(1-\beta_{2}\right)^{n}+\beta_{3}^{n} .
\end{aligned}
$$

Therefore, for $1 \leq r_{1} \leq n-1$ and $1 \leq r_{2} \leq n-r_{1}$, we get

$$
P\left(R_{1}=r_{1}, R_{2}=r_{2} \mid A\right)=\frac{c_{r_{1}, r_{2}} \beta_{1}^{r_{1}} \beta_{2}^{r_{2}} \beta_{3}^{n-r_{1}-r_{2}}}{1-\left(1-\beta_{1}\right)^{n}-\left(1-\beta_{2}\right)^{n}+\beta_{3}^{n}} .
$$

Using (3.2), we get

$$
P\left(R_{1}=r_{1} \mid A\right)=\left(\begin{array}{c}
n \\
r_{1}
\end{array}\right) \frac{\beta_{1}^{r_{1}}\left(\left(1-\beta_{1}\right)^{n-r_{1}}-\beta_{3}^{n-r_{1}}\right)}{1-\left(1-\beta_{1}\right)^{n}-\left(1-\beta_{2}\right)^{n}+\beta_{3}^{n}}
$$

and

$$
P\left(R_{2}=r_{2} \mid R_{1}=r_{1}, A\right)=\left(\begin{array}{c}
n-r_{1} \\
r_{2}
\end{array}\right)\left(\frac{\beta_{2}}{1-\beta_{1}}\right)^{r_{2}}\left(1-\frac{\beta_{2}}{1-\beta_{1}}\right)^{n-r_{1}-r_{2}} \times \frac{1-\beta_{1}^{n}-\left(1-\beta_{1}\right)^{n}}{1-\left(1-\beta_{1}\right)^{n}-\left(1-\beta_{2}\right)^{n}+\beta_{3}^{n}} .
$$

Moreover, by performing some algebraic calculations, it is deduced that given $A$, the conditional expected value of $R_{1}$ is as follows

$$
E_{\theta_{1}, \theta_{2}}\left(R_{1} \mid A\right)=\frac{n \beta_{1}\left(1-\left(1-\beta_{2}\right)^{n-1}\right)}{1-\left(1-\beta_{1}\right)^{n}-\left(1-\beta_{2}\right)^{n}+\beta_{3}^{n}},
$$


where $E_{\theta_{1}, \theta_{2}}$ denotes the fact that the expectation depends on $\theta_{1}$ and $\theta_{2}$. Similarly, we get

$$
E_{\theta_{1}, \theta_{2}}\left(R_{2} \mid A\right)=\frac{n \beta_{2}\left(1-\left(1-\beta_{1}\right)^{n-1}\right)}{1-\left(1-\beta_{1}\right)^{n}-\left(1-\beta_{2}\right)^{n}+\beta_{3}^{n}} .
$$

- The joint conditional distribution of $Z_{1}, Z_{2}, \ldots, Z_{w_{1}}$, given $R_{1}=r_{1}$, is multinomial with parameters $r_{1}, \pi_{1} / \sum_{i=1}^{w_{1}} \pi_{i}, \ldots, \pi_{w_{1}} / \sum_{i=1}^{w_{1}} \pi_{i}$, where $\pi_{i}$ is as defined in (2.3). So, it is easy to find the corresponding pmf is as follows

$$
P\left(Z_{1}=z_{1}, \ldots, Z_{w_{1}}=z_{w_{1}} \mid R_{1}=r_{1}\right)=\frac{r_{1} !}{\prod_{i=1}^{w_{1}} z_{i} !} \frac{p_{1}^{r_{1}} q_{1}^{\sum_{i=1}^{w_{1}} i z_{i}-r_{1}}}{\left(1-q_{1}^{w_{1}}\right)^{r_{1}}} .
$$

- The joint conditional distribution of $Z_{w_{1}+1}, \ldots, Z_{w_{2}}$, given $R_{2}=r_{2}$, is multinomial with parameters $r_{2}, \pi_{w_{1}+1} / \sum_{i=w_{1}+1}^{w_{2}} \pi_{i}, \ldots, \pi_{w_{2}} / \sum_{i=w_{1}+1}^{w_{2}} \pi_{i}$. It can be shown that

$$
P\left(Z_{w_{1}+1}=z_{w_{1}+1}, \ldots, Z_{w_{2}}=z_{w_{2}} \mid R_{2}=r_{2}\right)=\frac{r_{2} !}{\prod_{i=w_{1}+1}^{w_{2}} z_{i} !} \frac{p_{2}^{r_{2}} q_{2}^{\sum_{i=w_{1}+1}^{w_{2}} i z_{i}-\left(w_{1}+1\right) r_{2}}}{\left(1-q_{2}^{w_{2}-w_{1}}\right)^{r_{2}}} .
$$

Now, using the above results, we present conditional cdfs of $\hat{\theta}_{1}$ and $\hat{\theta}_{2}$ in the following theorems.

Theorem 1. The conditional cdf of $\hat{\theta}_{1}$, given $A$, is

$$
F_{\hat{\theta}_{1} \mid A}\left(x ; \theta_{1}, \theta_{2}\right)=\sum_{r_{1}=1}^{n-1} \sum_{r_{2}=1}^{n-r_{1}} \sum_{\mathfrak{I}_{1}\left(r_{1} x-w_{1}\left(n-r_{1}\right)\right)} \frac{r_{1} ! c_{r_{1}, r_{2}}}{\prod_{i=1}^{w_{1}} z_{i} !} \frac{\beta_{2}^{r_{2}} \beta_{3}^{n-r_{1}-r_{2}} p_{1}^{r_{1}} q_{1}^{\sum_{i=1}^{w_{1}} i_{i}-r_{1}}}{1-\left(1-\beta_{1}\right)^{n}-\left(1-\beta_{2}\right)^{n}+\beta_{3}^{n}},
$$

where

$$
\mathfrak{J}_{1}(x)=\left\{\left(z_{1}, \ldots, z_{w_{1}}\right): \sum_{i=1}^{w_{1}} z_{i}=r_{1}, \sum_{i=1}^{w_{1}} i z_{i} \leq x\right\}
$$

Proof: Notice that the conditional cdf of $\hat{\theta}_{1}$, given $A$, can be written as follows

$$
\begin{aligned}
F_{\hat{\theta}_{1} \mid A}(x) & =P\left(\hat{\theta}_{1} \leq x \mid A\right) \\
& =\sum_{r_{1}=1}^{n-1} \sum_{r_{2}=1}^{n-r_{1}} P\left(\hat{\theta}_{1} \leq x \mid R_{1}=r_{1}, R_{2}=r_{2}\right) P\left(R_{1}=r_{1}, R_{2}=r_{2} \mid A\right) .
\end{aligned}
$$

Furthermore, from (2.5) and (3.7), we find

$$
\begin{aligned}
P\left(\hat{\theta}_{1} \leq x \mid R_{1}=r_{1}, R_{2}=r_{2}\right) & =P\left(\sum_{i=1}^{w_{1}} i Z_{i} \leq r_{1} x-w_{1}\left(n-r_{1}\right) \mid R_{1}=r_{1}\right) \\
& =\sum_{\mathfrak{J}_{1}\left(r_{1} x-w_{1}\left(n-r_{1}\right)\right)} \frac{r_{1} !}{\prod_{i=1}^{w_{1}} z_{i} !} \frac{p_{1}^{r_{1}} q_{1}^{\sum_{i=1}^{w_{1}} i z_{i}-r_{1}}}{\left(1-q_{1}^{w_{1}}\right)^{r_{1}}},
\end{aligned}
$$


where the summation index $\mathfrak{J}_{1}(x)$ extends over all integers $z_{1}, \ldots, z_{w_{1}}$ for which $\sum_{i=1}^{w_{1}} z_{i}=r_{1}$ and $\sum_{i=1}^{w_{1}} i z_{i} \leq x$. Substituting (3.2) and (3.12) in (3.11), the result follows.

Theorem 2. The conditional cdf of $\hat{\theta}_{2}$, given $A$, is

$$
\begin{aligned}
F_{\hat{\theta}_{2} \mid A}\left(x ; \theta_{1}, \theta_{2}\right)= & \sum_{r_{1}=1}^{n-1} \sum_{r_{2}=1}^{n-r_{1}} \sum_{\mathfrak{J}_{2}(b(x))} \frac{r_{2} ! c_{r_{1}, r_{2}}}{\prod_{i=w_{1}+1}^{w_{2}} z_{i} !} \frac{\beta_{1}^{r_{1}} \beta_{3}^{n-r_{1}-r_{2}}}{1-\left(1-\beta_{1}\right)^{n}-\left(1-\beta_{2}\right)^{n}+\beta_{3}^{n}} \\
& \times\left(q_{1}^{w_{1}} p_{2}\right)^{r_{2}} q_{2}^{\sum_{i=w_{1}+1}^{w_{2}} i z_{i}-r_{2}\left(w_{1}+1\right)},
\end{aligned}
$$

where $b(x)=r_{2} x-\left(w_{2}-w_{1}\right)\left(n-r_{1}-r_{2}\right)+w_{1} r_{2}$ and

$$
\mathfrak{J}_{2}(x)=\left\{\left(z_{w_{1}+1}, \ldots, z_{w_{2}}\right): \sum_{i=w_{1}+1}^{w_{2}} z_{i}=r_{2}, \sum_{i=w_{1}+1}^{w_{2}} i z_{i} \leq x\right\} .
$$

Proof: Using (2.6) and (3.8), analogous to proof of Theorem 1, the result deduces.

\section{Confidence intervals}

In this section, we use different methods to construct CIs for the unknown parameters $\theta_{1}$ and $\theta_{2}$.

\subsection{Exact Cls}

Suppose that the tail probability of $\hat{\theta}_{k}$, i.e., $\mathrm{P}_{\theta_{k}}\left(\hat{\theta}_{k}>\xi\right)$, is monotone increasing function of $\theta_{k}(k=1,2)$. If $\hat{\theta}_{k}^{\text {obs }}$ denotes the observed value of $\hat{\theta}_{k}$, then $\left(\theta_{k}^{L}, \theta_{k}^{U}\right)$ is an exact $100(1-\alpha) \%$ CI for $\theta_{k}$, where $\theta_{k}^{L}$ and $\theta_{k}^{U}$ can be obtained by solving the equations

$$
\mathrm{P}_{\theta_{k}^{L}}\left(\hat{\theta}_{k}>\hat{\theta}_{k}^{\mathrm{obs}}\right)=\frac{\alpha}{2} \quad \text { and } \quad \mathrm{P}_{\theta_{k}^{U}}\left(\hat{\theta}_{k}>\hat{\theta}_{k}^{\mathrm{obs}}\right)=1-\frac{\alpha}{2} .
$$

This approach has been applied to construct exact CI in different contexts by several authors. See, for example, Balakrishnan and Han (2008), Balakrishnan and Xie (2007a, 2007b), Balakrishnan et al. (2009), Chen and Bhattacharyya (1988), Childs et al. (2003), Gupta and Kundu (1998), and Kundu and Basu (2000). Further, the required monotonicity in this approach follows those of Balakrishnan and Iliopoulos (2010). According to the above scenario, the interval $\left(\theta_{1}^{L}, \theta_{1}^{U}\right)$ is the exact $100(1-\alpha) \%$ CI for $\theta_{1}$, if the following two non-linear equations hold

$$
\left\{\begin{array}{l}
1-\frac{\alpha}{2}=F_{\hat{\theta}_{1} \mid A}\left(\hat{\theta}_{1}^{\mathrm{obs}} ; \theta_{1}^{L}, \hat{\theta}_{2}^{\mathrm{obs}}\right), \\
\frac{\alpha}{2}=F_{\hat{\theta}_{1} \mid A}\left(\hat{\theta}_{1}^{\mathrm{obs}} ; \theta_{1}^{U}, \hat{\theta}_{2}^{\mathrm{obs}}\right),
\end{array}\right.
$$

where $F_{\hat{\theta}_{1} \mid A}\left(x ; \theta_{1}, \theta_{2}\right)$ is as defined in (3.9). Similarly, $\left(\theta_{2}^{L}, \theta_{2}^{U}\right)$ is the exact $100(1-\alpha) \%$ CI for $\theta_{2}$, if

$$
\left\{\begin{array}{l}
1-\frac{\alpha}{2}=F_{\hat{\theta}_{2} \mid A}\left(\hat{\theta}_{2}^{\mathrm{obs}} ; \hat{\theta}_{1}^{\mathrm{obs}}, \theta_{2}^{L}\right), \\
\frac{\alpha}{2}=F_{\hat{\theta}_{2} \mid A}\left(\hat{\theta}_{2}^{\mathrm{obs}} ; \hat{\theta}_{1}^{\mathrm{obs}}, \theta_{2}^{U}\right),
\end{array}\right.
$$

where $F_{\hat{\theta}_{2} \mid A}\left(x ; \theta_{1}, \theta_{2}\right)$ is as defined in (3.13). 


\subsection{Approximate $\mathrm{Cl}$}

Consider a random sample of size $n$ from the cdf in (2.2) and let $\hat{\theta}_{k}$ be the MLE of $\theta_{k}(k=1,2)$, then for large $n$, an approximate $100(1-\alpha) \%$ CI for $\theta_{k}$ is given by

$$
\hat{\theta}_{k}-z_{1-\frac{\alpha}{2}} \sqrt{\frac{1}{\hat{I}_{k}\left(\theta_{1}, \theta_{2}\right)}} \leq \theta_{k} \leq \hat{\theta}_{k}+z_{1-\frac{\alpha}{2}} \sqrt{\frac{1}{\hat{I}_{k}\left(\theta_{1}, \theta_{2}\right)}}, \quad k=1,2,
$$

where $\hat{I}_{k}\left(\theta_{1}, \theta_{2}\right)$ is the approximate Fisher information (FI) about $\theta_{k}$. In this section, we use two approaches to evaluate $\hat{I}_{k}\left(\theta_{1}, \theta_{2}\right)$.

Approach I. Observed FI is a reasonable approximation for the expected FI in (4.3) (see Casella and Berger, 1990, p. 326). Therefore, we use the following approximation to construct CI for $\theta_{k}$ :

$$
\hat{I}_{k}\left(\theta_{1}, \theta_{2}\right)=-\left.\frac{\partial^{2}}{\partial \theta_{k}^{2}} \ell\left(\theta_{1}, \theta_{2}\right)\right|_{\theta_{1}=\hat{\theta}_{1}, \theta_{2}=\hat{\theta}_{2}},
$$

where $\ell\left(\theta_{1}, \theta_{2}\right)$ is the log-likelihood function of $\theta_{1}$ and $\theta_{2}$, which is obtained by taking the logarithm of the pmf in (2.4), when the reparameterization $p_{i}=1 / \theta_{i}(i=1,2)$ is performed. By doing some algebraic calculations it can be shown that

$$
\hat{I}_{1}\left(\theta_{1}, \theta_{2}\right)=\frac{R_{1}}{\hat{\theta}_{1}\left(\hat{\theta}_{1}-1\right)} \quad \text { and } \quad \hat{I}_{2}\left(\theta_{1}, \theta_{2}\right)=\frac{R_{2}}{\hat{\theta}_{2}\left(\hat{\theta}_{2}-1\right)} .
$$

The approximate CIs obtained from this approach are denoted by Appr-I CIs in the sequel.

Approach II. Another approximation for the expected FI can be derived by substituting $\hat{\theta}_{k}$ instead of $\theta_{k}(k=1,2)$ in the associated quantity, i.e.,

$$
\hat{I}_{k}\left(\theta_{1}, \theta_{2}\right)=\left.E\left(-\frac{\partial^{2}}{\partial \theta_{k}^{2}} \ell\left(\theta_{1}, \theta_{2}\right)\right)\right|_{\theta_{1}=\hat{\theta}_{1}, \theta_{2}=\hat{\theta}_{2}} .
$$

By performing some algebraic calculations, we have

$$
\hat{I}_{1}\left(\theta_{1}, \theta_{2}\right)=\frac{2 \hat{\theta}_{1}-1}{\hat{\theta}_{1}^{2}\left(\hat{\theta}_{1}-1\right)^{2}}\left\{\left(\hat{\theta}_{1}-\frac{w_{1}\left(\hat{\theta}_{1}-1\right)^{w_{1}}}{\hat{\theta}_{1}^{w_{1}}-\left(\hat{\theta}_{1}-1\right)^{w_{1}}}-\left(w_{1}+1\right)\right) E_{\hat{\theta}_{1}, \hat{\theta}_{2}}\left(R_{1} \mid A\right)+n w_{1}\right\}-\frac{1}{\hat{\theta}_{1}^{2}} E_{\hat{\theta}_{1}, \hat{\theta}_{2}}\left(R_{1} \mid A\right)
$$

and

$$
\begin{aligned}
\hat{I}_{2}\left(\theta_{1}, \theta_{2}\right)= & \frac{2 \hat{\theta}_{2}-1}{\hat{\theta}_{2}^{2}\left(\hat{\theta}_{2}-1\right)^{2}}\left\{\left(\hat{\theta}_{2}-\frac{\left(w_{2}-w_{1}\right)\left(\hat{\theta}_{2}-1\right)^{w_{2}-w_{1}}}{\hat{\theta}_{2}^{w_{2}-w_{1}}-\left(\hat{\theta}_{2}-1\right)^{w_{2}-w_{1}}}-1\right) E_{\hat{\theta}_{1}, \hat{\theta}_{2}}\left(R_{2} \mid A\right)+\left(w_{2}-w_{1}\right) E_{\hat{\theta}_{1}, \hat{\theta}_{2}}\left(n-R_{1}-R_{2} \mid A\right)\right\} \\
& -\frac{1}{\hat{\theta}_{2}^{2}} E_{\hat{\theta}_{1}, \hat{\theta}_{2}}\left(R_{2} \mid A\right)
\end{aligned}
$$

where $E_{\theta_{1}, \theta_{2}}\left(R_{1} \mid A\right)$ and $E_{\theta_{1}, \theta_{2}}\left(R_{2} \mid A\right)$ are as defined in (3.5) and (3.6), respectively.

Hereinafter, we show the approximate CIs obtained from this approach by the Appr-II CIs. 


\subsection{Bootstrap Cls}

Here, we present several bootstrap methods to construct CIs for $\theta_{1}$ and $\theta_{2}$. Toward this end, we use the following resampling algorithm for given $n, w_{1}$ and $w_{2}$ :

Step 1. Using the original Type-I censored sample, we obtain $\hat{\theta}_{1}$ and $\hat{\theta}_{2}$ from the equations in (2.5) and (2.6), respectively.

Step 2. By substituting $\hat{\theta}_{1}$ and $\hat{\theta}_{2}$ in the conditional pmf (3.3), a value for $R_{1}$ is generated.

Step 3. For obtained $R_{1}=r_{1}$, by substituting $\hat{\theta}_{1}$ and $\hat{\theta}_{2}$ in (3.4), a value for $R_{2}$ is generated.

Step 4. Based on the observed values of $R_{1}=r_{1}$ and $R_{2}=r_{2}$, the random samples $\left(Z_{1}, \ldots, Z_{w_{1}}\right)$ and $\left(Z_{w_{1}+1}, \ldots, Z_{w_{1}+w_{2}}\right)$ are generated from (3.7) and (3.8), respectively.

Step 5. The estimators $\hat{\theta}_{1}^{*}$ and $\hat{\theta}_{2}^{*}$ are derived form (2.5) and (2.6), respectively.

Step 6. Repeat Steps $2-5, M$ times and arrange all $\hat{\theta}_{1}^{*}$ 's and $\hat{\theta}_{2}^{*}$ 's in ascending order to obtain the bootstrap sample

$$
\left\{\hat{\theta}_{k}^{*(1)}, \hat{\theta}_{k}^{*(2)}, \ldots, \hat{\theta}_{k}^{*(M)}\right\}, \quad k=1,2
$$

Using the above algorithm, we can construct some bootstrap CIs in different methods that are studied in the sequel. For more details one may refer to Efron and Tibshirani (1993).

\subsubsection{Percentile interval}

Using the bootstrap samples presented in (4.4), a two-sided $100(1-\alpha) \%$ percentile bootstrap CI for $\theta_{k}$ is given by

$$
\left(\hat{\theta}_{k}^{*\left(\frac{\alpha M}{2}\right)}, \hat{\theta}_{k}^{*\left(\left(1-\frac{\alpha}{2}\right) M\right)}\right)
$$

If $\alpha M / 2$ or $(1-\alpha / 2) M$ in (4.5) are not integer, we use the integer parts of $(\alpha / 2)(M+1)$ or $(1-\alpha / 2)(M+$ $1)$, respectively. Note that the CI in (4.5) is an equi-tailed CI. The shortest width $100(1-\alpha) \%$ percentile bootstrap CI is

$$
\left(\hat{\theta}_{k}^{* L}, \hat{\theta}_{k}^{* U}\right)
$$

where it is obtained by considering all possible $100(1-\alpha) \%$ CIs of the form

$$
\left(\hat{\theta}_{k}^{*(i)}, \hat{\theta}_{k}^{*((1-\alpha) M+i)}\right), \quad i=1, \ldots, \alpha M, k=1,2,
$$

and choosing the interval with minimum width.

In the next sections, the notations $\mathrm{P}$ and ShWP will show the percentile and shortest width percentile bootstrap CIs, respectively. 


\subsubsection{Studentized-t interval}

Here, we use the statistic

$$
T_{k}^{*(j)}=\frac{\hat{\theta}_{k}^{*(j)}-\hat{\theta}_{k}}{\widehat{\operatorname{se}}\left(\hat{\theta}_{k}^{*}\right)}, \quad j=1, \ldots, M, \quad k=1,2,
$$

where $\widehat{\operatorname{se}}\left(\hat{\theta}_{k}^{*}\right)$ is the estimated standard error of $\hat{\theta}_{k}^{*}$ for the bootstrap samples. A two-sided equi-tailed $100(1-\alpha) \%$ studentized- $t$ (St) bootstrap CI for $\theta_{k}$ is

$$
\left(\hat{\theta}_{k}-T_{k}^{*\left(\left(1-\frac{\alpha}{2}\right) M\right)} \widehat{\operatorname{se}}\left(\hat{\theta}_{k}\right), \hat{\theta}_{k}-T_{k}^{*\left(\frac{\alpha M}{2}\right)} \widehat{\operatorname{se}}\left(\hat{\theta}_{k}\right)\right),
$$

where $\widehat{\operatorname{se}}\left(\hat{\theta}_{k}\right)$ can be obtained from the asymptotic variance of the original Type-I censored sample. That is, $\widehat{\operatorname{se}}\left(\hat{\theta}_{k}\right) \approx \sqrt{1 / \hat{I}_{k}\left(\theta_{1}, \theta_{2}\right)}$, where the expected FI can be approximated via Approaches I and II, presented in Subsection 4.2. For the corresponding St bootstrap CIs, we shall use the notations St-I and St-II, respectively, in the next sections. Moreover, the shortest width $100(1-\alpha) \%$ St bootstrap CI is

$$
\left(\hat{\theta}_{k}-T_{k}^{* U} \widehat{\operatorname{se}}\left(\hat{\theta}_{k}\right), \hat{\theta}_{k}-T_{k}^{* L} \widehat{\operatorname{se}}\left(\hat{\theta}_{k}\right)\right),
$$

where $\left(T_{k}^{* L}, T_{k}^{* U}\right)$ has the minimum width among all possible $100(1-\alpha) \%$ CIs of the form

$$
\left(T_{k}^{*(i)}, T_{k}^{*((1-\alpha) M+i)}\right), \quad i=1, \ldots, \alpha M, k=1,2 .
$$

If Approach I or II is used to approximate $\widehat{\operatorname{se}}\left(\hat{\theta}_{k}\right)$ in (4.8), the corresponding shortest width bootstrap CIs are denoted by ShWSt-I or ShWSt-II, respectively.

\section{Simulation study}

To compare the proposed confidence intervals in terms of coverage probability $(\mathrm{CP})$ and expected width (EW), a simulation study has been conducted using the following algorithm:

1. For given values of $n, w_{1}, w_{2}, p_{1}$ and $p_{2}$, the value of $R_{1}$ is generated by use of the (3.3).

2. Based on the observed value of $R_{1}=r_{1}$, the value of $\left(Z_{1}, \ldots, Z_{w_{1}}\right)$ is simulated from (3.7); then the MLE of $\theta_{1}$ is derived using (2.5).

3. Given $R_{1}=r_{1}$, an observation is generated from the conditional pmf (3.4) to obtain the observed value of $R_{2}$

4. Given $R_{2}=r_{2}$, the value of $\left(Z_{w_{1}+1}, \ldots, Z_{w_{2}}\right)$ is extracted from (3.8); then the value of $\hat{\theta}_{2}$ is obtained using (2.6).

5. For given $\alpha$, the exact CIs for $\theta_{1}$ and $\theta_{2}$ are derived by solving the equations in (4.1) and (4.2), respectively. The Appr-I and Appr-II CIs for $\theta_{k}(k=1,2)$ are obtained using (4.3).

6. To derive the various bootstrap CIs, the bootstrap samples are generated using Steps 2-6 of the algorithm presented in Section 4.3. The percentiles and ShWP bootstrap CIs are then derived using (4.5) and (4.6), respectively. The St-I and St-II bootstrap CIs are obtained using (4.7). ShWSt-I and ShWSt-II bootstrap CIs are also constructed using (4.8). 
7. The Steps $1-6$ are repeated $B$ times to attain the CPs and EWs of various CIs.

Table 1 represents the results for $n=10,15,20, p_{1}=0.1, p_{2}=0.2, w_{1}=5, w_{2}=10, \alpha=0.05,0.1$ and $B=M=1000$. From Table 1, for given $n$, it is deduced that:

- Based on minimum EW criterion, ShWSt-I bootstrap CIs may be suggested for both of $\theta_{1}$ and $\theta_{2}$.

- In view of closeness the CP to the nominal level, the exact CIs are offered for both of $\theta_{1}$ and $\theta_{2}$, but, the EWs of these CIs are too large.

- Considering both criteria EW and CP, it is reasonable to choose the CI with minimum EW among those with CPs near to the nominal level. Therefore, one may suggest the percentile bootstrap CIs or exact CIs to estimate both of $\theta_{1}$ and $\theta_{2}$. Note that the EW of exact CIs is excessively larger than the percentile bootstrap CIs for $n=10$, whereas there is no significant difference for larger samples.

- Comparing between performance the Approaches I and II in approximating the FI, it is observed that using Approach I leads to shorter EW than Approach II for approximate CIs as well as studentized bootstrap CIs for both of $\theta_{1}$ and $\theta_{2}$.

- When $n$ increases, the EWs of all CIs decrease.

\section{Illustrative example}

To illustrate the proposed procedure in this paper, we consider a numerical example. Assuming $w_{1}=5$ and $w_{2}=10$, a random ample of size 20 has been generated from the cdf in (2.2) with $p_{1}=0.1$ and $p_{2}=0.2$. Equivalently, we get $\theta_{1}=10$ and $\theta_{2}=5$. Table 2 presents the generated data.

Using the data in Table 2, we would obtain that $R_{1}=8$ and $R_{2}=9$ are the number of units failed at stress level $s_{0}$ and $s_{1}$, respectively. Using (2.5) and (2.6), the MLEs have been evaluated as $\hat{\theta}_{1}=10.25$ and $\hat{\theta}_{2}=4$, respectively.

We only derive exact CIs, percentile and ShWSt-I bootstrap CIs, because the results of Section 5 show that these CIs are more important than the others. For given $\alpha$, the exact CIs for $\theta_{1}$ and $\theta_{2}$ are derived by solving the equations in (4.1) and (4.2), respectively. We use $M=1000$ bootstrap samples to construct the bootstrap CIs. The percentile and ShWSt-I bootstrap CIs are obtained using (4.5) and (4.8), respectively. Table 3 tabulates the results for significant levels $\alpha=0.05,0.10$. It, deduces that:

1. All derived CIs contain the exact values of parameters.

2. As mentioned in Section 5, the ShWSt-I bootstrap CIs for both of $\theta_{1}$ and $\theta_{2}$ have shorter width than other CIs.

3. The exact CIs for both of $\theta_{1}$ and $\theta_{2}$ have larger width than other CIs; however, the results in Section 5 indicate that they have more accurate CPs.

\section{Conclusions}

This paper considered a simple step-stress model with Type-I censored samples from geometric distribution. The MLEs of the means of geometric distributions in both levels of stress, $\theta_{1}$ and $\theta_{2}$, were determined and the associated distributions derived. Several methods were used to construct CIs for 
Table 1: The simulated CPs and EWs for various CIs of $\theta_{1}$ and $\theta_{2}$

\begin{tabular}{|c|c|c|c|c|c|c|c|}
\hline \multirow{2}{*}{ Parameter } & \multirow{2}{*}{ Method } & \multicolumn{3}{|c|}{$90 \%$} & \multicolumn{3}{|c|}{$95 \%$} \\
\hline & & $n=10$ & $n=15$ & $n=20$ & $n=10$ & $n=15$ & $n=20$ \\
\hline \multirow{18}{*}{$\theta_{1}$} & \multirow{2}{*}{ Bootstrap (P) } & $25.1614^{*}$ & 23.3402 & 18.4297 & 31.5360 & 29.3901 & 23.9198 \\
\hline & & $0.9057^{* *}$ & 0.8776 & 0.8667 & 0.9358 & 0.9426 & 0.9283 \\
\hline & \multirow{2}{*}{ Bootstrap (ShWP) } & 22.0043 & 18.1397 & 14.8762 & 26.4382 & 24.9321 & 20.0466 \\
\hline & & 0.7794 & 0.8585 & 0.8500 & 0.9038 & 0.9006 & 0.9217 \\
\hline & \multirow{2}{*}{ Bootstrap (St-I) } & 18.4873 & 12.5858 & 11.0341 & 19.9077 & 14.5434 & 13.4059 \\
\hline & & 0.6573 & 0.7725 & 0.7667 & 0.6901 & 0.7992 & 0.7967 \\
\hline & \multirow{2}{*}{ Bootstrap (ShWSt-I) } & 17.0203 & 10.5830 & 9.2594 & 19.0453 & 13.3769 & 12.0078 \\
\hline & & 0.7023 & 0.8011 & 0.7600 & 0.7243 & 0.8337 & 0.8267 \\
\hline & \multirow{2}{*}{ Bootstrap (St-II) } & 21.3086 & 12.6408 & 11.0573 & 22.8299 & 14.5997 & 13.4333 \\
\hline & & 0.6595 & 0.7667 & 0.7683 & 0.6880 & 0.7954 & 0.7933 \\
\hline & \multirow{2}{*}{ Bootstrap (ShWSt-II) } & 19.6041 & 10.6452 & 9.2820 & 21.8643 & 13.4350 & 12.0320 \\
\hline & & 0.6959 & 0.8050 & 0.7617 & 0.7264 & 0.8317 & 0.8300 \\
\hline & \multirow{2}{*}{ Appr-I } & 23.2292 & 16.3139 & 13.8510 & 26.5624 & 19.1938 & 16.4145 \\
\hline & & 0.8736 & 0.8948 & 0.8717 & 0.9166 & 0.9273 & 0.9067 \\
\hline & \multirow{2}{*}{ Appr-II } & 25.2416 & 16.4659 & 13.9088 & 28.9052 & 19.3378 & 16.4688 \\
\hline & & 0.8736 & 0.8948 & 0.8717 & 0.9038 & 0.9254 & 0.9067 \\
\hline & \multirow{2}{*}{ Exact } & 43.9552 & 23.0489 & 17.1317 & 54.4006 & 30.2843 & 22.0887 \\
\hline & & 0.8951 & 0.9025 & 0.8883 & 0.9487 & 0.9541 & 0.9433 \\
\hline \multirow{18}{*}{$\theta_{2}$} & \multirow{2}{*}{ Bootstrap (P) } & 11.7757 & 9.5233 & 7.5525 & 15.0301 & 12.6284 & 9.9035 \\
\hline & & 0.8779 & 0.8757 & 0.8917 & 0.9188 & 0.8776 & 0.9450 \\
\hline & \multirow{2}{*}{ Bootstrap (ShWP) } & 9.8730 & 8.0076 & 6.5239 & 12.8614 & 10.6132 & 8.5210 \\
\hline & & 0.8415 & 0.8509 & 0.8800 & 0.9102 & 0.8585 & 0.9367 \\
\hline & \multirow{2}{*}{ Bootstrap (St-I) } & 8.7932 & 6.4302 & 5.3088 & 9.5912 & 7.5541 & 6.5471 \\
\hline & & 0.7131 & 0.7438 & 0.7800 & 0.7414 & 0.7725 & 0.8233 \\
\hline & \multirow{2}{*}{ Bootstrap (ShWSt-I) } & 8.3315 & 5.6958 & 4.7262 & 9.5671 & 7.1394 & 5.9811 \\
\hline & & 0.7537 & 0.7954 & 0.8233 & 0.7799 & 0.8011 & 0.8500 \\
\hline & \multirow{2}{*}{ Bootstrap (St-II) } & 9.5278 & 6.7310 & 5.4697 & 10.3522 & 7.8479 & 6.7123 \\
\hline & & 0.7109 & 0.7476 & 0.7817 & 0.7521 & 0.7667 & 0.8217 \\
\hline & \multirow{2}{*}{ Bootstrap (ShWSt-II) } & 9.1996 & 6.0236 & 4.9110 & 10.3318 & 7.4575 & 6.1503 \\
\hline & & 0.7644 & 0.7954 & 0.8267 & 0.7763 & 0.8050 & 0.8533 \\
\hline & \multirow{2}{*}{ Appr-I } & 10.3003 & 7.6594 & 6.2390 & 11.8528 & 9.0149 & 7.3936 \\
\hline & & 0.8629 & 0.8604 & 0.8817 & 0.8867 & 0.8948 & 0.9150 \\
\hline & \multirow{2}{*}{ Appr-II } & 11.0150 & 7.9019 & 6.3693 & 12.6716 & 9.3000 & 7.5452 \\
\hline & & 0.8651 & 0.8585 & 0.8833 & 0.8974 & 0.8948 & 0.9150 \\
\hline & \multirow{2}{*}{ Exact } & 31.9176 & 12.9558 & 8.2693 & 46.6476 & 17.1537 & 10.6431 \\
\hline & & 0.9058 & 0.9101 & 0.9167 & 0.9487 & 0.9025 & 0.9583 \\
\hline
\end{tabular}

$\overline{\mathrm{CP}}=$ coverage probability; $\mathrm{EW}=$ expected width; $\mathrm{CI}=$ confidence interval. ${ }^{*}$ and ${ }^{* *}$ stand for the EWs and CPs of the CIs.

Table 2: Generated sample of size 20 from the cdf in (2.2) with $w_{1}=5, w_{2}=10, \theta_{1}=10$, and $\theta_{2}=5$

\begin{tabular}{clllllllll}
\hline \hline Mean failure time & \multicolumn{10}{c}{ Failure times } \\
\hline$\theta_{1}=10$ & 1 & 2 & 2 & 2 & 2 & 3 & 5 & 5 & \\
$\theta_{2}=5$ & 6 & 6 & 6 & 6 & 7 & 8 & 9 & 9 & 9 \\
\hline \hline
\end{tabular}

$\theta_{1}$ and $\theta_{2}$ and they were compared via a simulation study. The ShWSt-I bootstrap CIs for both of $\theta_{1}$ and $\theta_{2}$ have shortest width; however, the CPs are significantly different from nominal levels. These CIs are derived using the asymptotic property of the MLEs; therefore, it is recommended to use them in the case of large sample sizes. Exact CIs can be extensively used for each sample size with satis- 
Table 3: CIs for $\theta_{1}$ and $\theta_{2}$ based on the data in Table 1 for $w_{1}=5$ and $w_{2}=10$

\begin{tabular}{clcc}
\hline \hline Parameter & Method & $90 \%$ & $95 \%$ \\
\hline \multirow{2}{*}{$\theta_{1}$} & Bootstrap (P) & $(6.4167,21.2500)$ & $(6.0000,23.7500)$ \\
& Bootstrap (ShWSt-I) & $(5.0874,13.2701)$ & $(2.7283,14.1818)$ \\
& Exact & $(6.2697,19.0887)$ & $(5.7711,21.8869)$ \\
\hline \multirow{2}{*}{$\theta_{2}$} & Bootstrap (P) & $(2.5000,6.8333)$ & $(2.3077,7.8000)$ \\
& Bootstrap (ShWSt-I) & $(2.0909,5.4656)$ & $(1.5959,5.8182)$ \\
\hline \hline
\end{tabular}

factory CPs; however, the required computation becomes difficult for large sample sizes. Hence, our recommendation is to apply ShWSt-I bootstrap CIs in the case of large sample sizes, when minimum $\mathrm{EW}$ is the only criterion to select the best CI. Exact CIs may be presented, when only the CP criterion is considered to choose the optimal CI. Percentile bootstrap CIs or exact CIs are suggested to estimate $\theta_{1}$ and $\theta_{2}$ in situations in which both of EW and CP criteria are important.

\section{References}

Arefi A and Razmkhah M (2013). Optimal simple step-stress plan for Type-I censored data from geometric distribution, Journal of the Iranian Statistical Society (JIRSS), 12, 193-210.

Arefi A, Razmkhah M, and Mohtashami Borzadaran GR (2011). Bayes estimation for a simple stepstress model with Type-I censored data from the geometric distribution, Journal of Statistical Research of Iran, 8, 149-169.

Bagdonavicius V and Nikulin M (2002). Accelerated Life Models: Modeling and Statistical Analysis, Chapman \& Hall/CRC Press, Boca Raton, FL.

Balakrishnan N, Cramer E, and Dembińska A (2011). Characterizations of geometric distribution through progressively Type-II right-censored order statistics, Statistics, 45, 559-573.

Balakrishnan N and Han D (2008). Exact inference for a simple step-stress model with competing risks for failure from exponential distribution under Type-II censoring, Journal of Statistical Planning and Inference, 138, 4172-4186.

Balakrishnan N and Iliopoulos G (2010). Stochastic monotonicity of the MLEs of parameters in exponential simple step-stress models under Type-I and Type-II censoring, Metrika, 72, 89-109.

Balakrishnan N and Xie Q (2007a). Exact inference for a simple step-stress model with Type-I hybrid censored data from the exponential distribution, Journal of Statistical Planning and Inference, 137, 3268-3290.

Balakrishnan N and Xie Q (2007b). Exact inference for a simple step-stress model with Type-II hybrid censored data from the exponential distribution, Journal of Statistical Planning and Inference, 137, 2543-2563.

Balakrishnan N, Xie Q, and Kundu D (2009). Exact inference for a simple step-stress model from the exponential distribution under time constraint, Annals of the Institute of Statistical Mathematics, 61, 251-274.

Casella G and Berger R (1990). Statistical Inference, Brooks/Cole Publishing, Pacific Grove, CA.

Chen SM and Bhattacharyya GK (1988). Exact confidence bound for an exponential parameter under hybrid censoring, Communication in Statistics-Theory and Methods, 17, 1858-1870.

Childs A, Chandrasekar B, Balakrishnan N, and Kundu D (2003). Exact likelihood inference based on Type-I and Type-II hybrid censored samples from the exponential distribution, Annals of the Institute of Statistical Mathematics, 55, 319-330.

Davarzani N and Parsian A (2011). Statistical inference for discrete middle-censored data, Journal of 
Statistical Planning and Inference, 141, 1455-1462.

Efron B and Tibshirani RJ (1993). An Introduction to the Bootstrap, Chapman \& Hall, New York.

Gupta RD and Kundu D (1998). Hybrid censoring with exponential failure distributions, Communication in Statistics-Theory and Methods, 27, 3065-3083.

Han D and Balakrishnan N (2010). Inference for a simple step-stress model with competing risks for failure from the exponential distribution under time constraint, Computational Statistics $\mathcal{E}$ Data Analysis, 54, 2066-2081.

Kundu D and Basu S (2000). Analysis of incomplete data in presence of competing risks, Journal of Statistical Planning and Inference, 87, 221-239.

Nelson W (1990). Accelerated Testing: Statistical Models, Test, Plans and Data Analyses, Wiley, New York.

Rezaei AH and Arghami NR (2002). Right censoring in a discrete life model, Metrika, 55, 151-160.

Wang R, Xu X, Pan R, and Sha N (2012). On parameter inference for step-stress accelerated life test with geometric distribution, Communication in Statistics-Theory and Methods, 41, 1796-1812. 